\title{
Modelling intestinal glucose absorption in premature infants using continuous glucose monitoring data
}

\author{
J. Knopp ${ }^{1 *}$, M. Signal ${ }^{1}$, D. Harris ${ }^{2,3}$, G Marics ${ }^{4}$, P. Weston ${ }^{2}$, J. Harding ${ }^{3}$, P.Tóth-Heyn ${ }^{4}$, J. \\ Hómlok $^{5}$, B. Benyó ${ }^{5}$, J.G Chase ${ }^{1}$
}

1. Department of Mechanical Engineering, University of Canterbury, Christchurch

2. Newborn Intensive Care Unit, Waikato District Health Board, Hamilton New Zealand

3. Liggins Institute, University of Auckland, Auckland, New Zealand

4. First Department of Paediatrics, Intensive Care Unit, Semmelweis University, Budapest, Hungary

5. Budapest University of Technology and Economics, Budapest, Hungary

${ }^{*}$ Corresponding author: Jennifer Dickson

Address:

Jennifer Knopp (nee Dickson)

Department of Mechanical Engineering

University of Canterbury

Private Bag 4800

Christchurch 8140

New Zealand

email: jennifer.dickson@canterbury.ac.nz

Phone: +64 33642987 exnt. 7650

\begin{tabular}{|l|l|l|}
\hline Name & First Affiliation & Email \\
\hline $\begin{array}{l}\text { Dr. Jennifer } \\
\text { Knopp }\end{array}$ & $\begin{array}{l}\text { University of Canterbury, New } \\
\text { Zealand }\end{array}$ & jennifer.dickson@canterbury.ac.nz \\
\hline $\begin{array}{l}\text { Dr. Matthew } \\
\text { Signal }\end{array}$ & $\begin{array}{l}\text { University of Canterbury, New } \\
\text { Zealand }\end{array}$ & Matthew.Signal@tirolifesciences.com \\
\hline $\begin{array}{l}\text { Dr. Deborah } \\
\text { Harris }\end{array}$ & $\begin{array}{l}\text { Waikato District Health Board, New } \\
\text { Zealand }\end{array}$ & Deborah.Harris@waikatodhb.health.nz \\
\hline Dr. Gábor Marics & Semmelweis University, Hungary & marics.gabor@gmail.com \\
\hline $\begin{array}{l}\text { Dr. Philip } \\
\text { Weston }\end{array}$ & $\begin{array}{l}\text { Waikato District Health Board, New } \\
\text { Zealand }\end{array}$ & phil.weston@waikatodhb.health.nz \\
\hline $\begin{array}{l}\text { Prof. Jane } \\
\text { Harding }\end{array}$ & $\begin{array}{l}\text { University of Auckland, New } \\
\text { Zealand }\end{array}$ & j.harding@auckland.ac.nz \\
\hline $\begin{array}{l}\text { Dr. Péter Tóth- } \\
\text { Heyn }\end{array}$ & Semmelweis University, Hungary & toth- \\
\hline $\begin{array}{l}\text { Mr. József } \\
\text { Homlok }\end{array}$ & $\begin{array}{l}\text { Budapest University of Technology } \\
\text { and Economics, Hungary }\end{array}$ & homlokjozsef@gmail.com \\
\hline $\begin{array}{l}\text { Prof. Balázs } \\
\text { Benyó }\end{array}$ & $\begin{array}{l}\text { Budapest University of Technology } \\
\text { and Economics, Hungary }\end{array}$ & bbenyo@iit.bme.hu \\
\hline $\begin{array}{l}\text { Prof. J. Geoffrey } \\
\text { Chase }\end{array}$ & $\begin{array}{l}\text { University of Canterbury, New } \\
\text { Zealand }\end{array}$ & geoff.chase@canterbury.ac.nz \\
\hline
\end{tabular}




\section{Abstract:}

Background: Model-based glycaemic control protocols have shown promise in neonatal intensive care units (NICUs) for reducing both hyperglycaemia and insulin-therapy driven hypoglycaemia. However, current models for the appearance of glucose from enteral feeding are based on values from adult intensive care cohorts. This study aims to determine enteral glucose appearance model parameters more reflective of premature infant physiology.

Methods: Peaks in CGM data associated with enteral milk feeds in preterm and term infants are used to fit a two compartment gut model. The first compartment describes glucose in the stomach, and the half life of gastric emptying is estimated as $\mathbf{2 0}$ minutes from literature. The second compartment describes glucose in the small intestine, and absorption of glucose into the blood is fit to CGM data. Two infant cohorts from two NICUs are used, and results are compared to appearances derived from data in highly controlled studies in literature.

Results: The average half life across all infants for glucose absorption from the gut to the blood was 50 minutes. This result was slightly slower than, but of similar magnitude to, results derived from literature. No trends were found with gestational or postnatal age. Breast milk fed infants were found to have a higher absorption constant than formula fed infants, a result which may reflect known differences in gastric emptying for different feed types.

Conclusions: This paper presents a methodology for estimation of glucose appearance due to enteral feeding, and model parameters suitable for a NICU model-based glycaemic control context.

Key Words: physiological modelling; glucose absorption; gut; glycemic control; premature infant; continuous glucose monitor 


\subsection{Introduction}

In neonatal intensive care hyperglycaemia is a common complication of prematurity $[1,2]$, and has been associated with worsened outcomes [2, 3, 4]. Postnatal growth restriction is a separate, but related, problem in premature infants and in extremely low birth weight (ELBW) infants [5]. The American Academy of Paediatrics suggests that nutrition goals should aim to achieve a postnatal growth rate approximating that of a normal foetus of the same gestational age $(G A)[6,7]$. This nutrition can be delivered via parenteral or enteral routes, and there is significant debate in literature around appropriate onset and clinical procedure for enteral feed initiation [8]. However, enhanced nutrition can potentiate and or exacerbate hyperglycaemia.

While it has been well-established that insulin therapy allows greater glucose tolerance and weight gain $[9,10,11,12,13]$, its effect on other clinical outcomes is not so well-defined. In particular, recent glycaemic control trials in the neonatal intensive care unit (NICU) have also suffered significant hypoglycaemia [14, 15]. Physiological model-based protocols offer great potential for allowing patient-specific care, including the ability to maximise or manage nutrition, and have demonstrated safe, effective control in adult and neonatal intensive care units $[16,17]$. However, model-based methods are only as good as the underlying models ability to capture inter- and intra- patient variability $[18,19,20]$.

Enteral feeding is a very common in many neonatal intensive care units, so a model of glucose appearance from enteral feeds is very necessary for model-based tight glycaemic control. Very little literature data exists directly quantifying the rate of glucose appearance from intestinal absorption in neonates, and is derived from extremely invasive studies involving short sections of intestine [21]. This study will develop a model of the appearance of glucose from enteral nutrition. This study does this in a minimally invasive manner using retrospective continuous glucose monitor (CGM) data in term and premature infants. In particular, the half-life of gastric absorption of glucose from the gut into 
the bloodstream is determined to better model glucose metabolism in the preterm neonate, and thus improve the safety and accuracy of glycaemic control.

\subsection{Methods}

\subsection{Two compartment gut model}

A simple two compartment model is used to model glucose transition through the digestive system

(Figure 1). The model comprises two compartments, $P_{1}$ and $P_{2}$, denoting glucose in the stomach and intestine, respectively. Glucose is delivered to the stomach as glucose, lactose, or another glucose based sugar, and is denoted $P_{e x}$. The amount [mmol] of glucose in the stomach, $\mathrm{P}_{1}$, is defined:

$$
\dot{P}_{1}=P_{e x}-d_{1} P_{1}
$$

Where $d_{1}[1 / \mathrm{min}]$ describes the rate of gastric emptying. The model thus captures the time delay of glucose appearance in the gut due to gastric emptying. Lactose is made up of glucose and galactose in roughly equal molar weight, and since galactose does not contribute directly to BG levels it was ignored. The amount of glucose in the gut, $P_{2}[\mathrm{mmol}]$, is defined:

$$
\dot{P}_{2}=d_{1} P_{1}-\min \left(P_{\text {max }}, d_{2} P_{2}\right)
$$

where $d_{2}[1 / \mathrm{min}]$ is the rate constants for glucose uptake from the gut into the blood, respectively. A supra-physiological upper limit from of $P_{\max }=6.0 \mathrm{mmol} / \mathrm{min}$ is applied $[22,23]$. This limit is taken from adult models of enteral feeding and glucose modelling [22] in the absence of data for this limit in neonates. Equation 2 is thus a function of rate parameters $d_{1}$ and $d_{2}$, which are not defined for this cohort

\subsection{The NICING glucose-insulin model for very premature infants}


The NICING (Neonatal Intensive Care Insulin-Nutrition-Glucose) model for glycaemic control in very/extremely preterm neonates is defined [24]:

$$
\begin{gathered}
\dot{G}=-p_{G} G(t)-S_{I} G(t) \frac{Q(t)}{1+\alpha_{G} Q(t)}+\frac{P_{E N}(t)+P_{E N}(t)+E G P * m_{\text {body }}-C N S * m_{\text {brain }}}{V_{g, f r a c}(t) * m_{\text {body }}} \\
\dot{I}=-\frac{n_{L} I(t)}{1+\alpha_{I} I(t)}-n_{K} I(t)-n_{I}(I(t)-Q(t))+\frac{u_{e x}(t)}{V_{P} * m_{\text {body }}}+\left(1-x_{L}\right) u_{e n} \\
\dot{Q}=n_{I} \frac{V_{P}}{V_{Q}}(I(t)-Q(t))-n_{C} \frac{Q(t)}{1+\alpha_{G} Q(t)} \\
u_{e n}= \begin{cases}\max (4.2,-1.5+1.9 * G) & \text { if female } \\
\max (2.2,-0.37+0.86 * G) & \text { if male }\end{cases}
\end{gathered}
$$

Parameter values and descriptions are given in Table 1, and the model is shown pictorially in Figure 1. All parameters are fixed, with the exception of $S_{I}$, which is time varying and fit on an hourly basis from clinical data using integral based fitting methods [25]. Further detail on the model and its origin can be found in [24] and the online supplement of [26]. This model is structurally similar to the ICING model for adults [22], and its dynamics are considered overall reflecting of human glucose-insulin dynamics. The NICING model will be used largely unchanged in this analysis for infants ranging from very preterm to term, with specific adaptions explained in the next section. It should be noted that Table 1 and $[24,26]$ present parameter values and models for central nervous system (CNS) uptake and the fluid volume of the interstitial space $\left(V_{Q}\right)$ which were originally developer for the extremely/very preterm infant (GA: 23 - 30 weeks). These aspects of the model will be updated to account for older infants in the next section. 


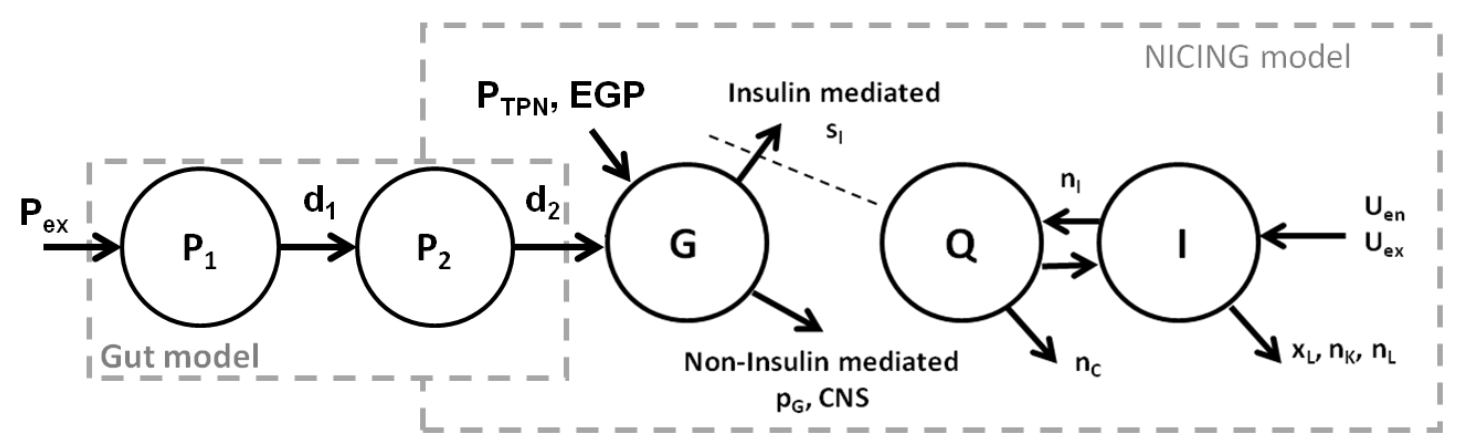

Figure 1: Pictorial representation of the Gut model and its interaction with the NICING pharmacodynamic model.

Table 1: NICING model parameter definitions and values [24].

\begin{tabular}{|c|c|c|}
\hline Parameter & Corresponding physiology & Value \\
\hline$p_{G}$ & Non-insulin mediated glucose uptake & $0.003 \mathrm{~min}^{-1}$ \\
\hline$S_{I}$ & Whole body insulin sensitivity & Variable $[\mathrm{L} / \mathrm{mU} / \mathrm{min}]$ \\
\hline$\alpha_{G}$ & Saturation on insulin-mediated glucose uptake & $0.0[\mathrm{~L} / \mathrm{mU}]$ \\
\hline$P_{T P N}(t)$ & Intravenous glucose delivery & Variable input [mmol/min] \\
\hline$P_{E N}(t)$ & Glucose absorption from gut & Variable input [mmol/min] \\
\hline$V_{g, f r a c}(t)$ & Distribution volume of glucose & $\mathrm{L} / \mathrm{kg}$ \\
\hline$E G P$ & Endogenous glucose production & $0.033 \mathrm{mmol} / \mathrm{kg} / \mathrm{min}$ \\
\hline CNS & Glucose uptake by central nervous system & $0.088 \mathrm{mmol} / \mathrm{kg} / \mathrm{min}$ \\
\hline$\alpha_{I}$ & Saturation on liver clearance of insulin & $0.0017 \mathrm{~L} / \mathrm{mU}$ \\
\hline$n_{L}$ & Liver clearance of Insulin & $0.39 \mathrm{~min}^{-1}$ \\
\hline$x_{L}$ & First pass liver clearance of insulin & 0.67 \\
\hline$n_{K}$ & Kidney clearance of insulin & $n_{K}=\frac{1}{V_{P}} \times \frac{0.9 G F R}{0.6}\left[\mathrm{~min}^{-1}\right]$ \\
\hline GFR & Glomerular Filtration Rate [27] & $G F R=0.45+0.24 m_{b w}+\frac{0.18 P N A}{7}$ \\
\hline$n_{I}$ & $\begin{array}{l}\text { Diffusion of insulin between plasma an } \\
\text { interstitial spaces }\end{array}$ & $0.025 \mathrm{~min}^{-1}$ \\
\hline$n_{C}$ & $\begin{array}{l}\text { Clearance and degradation of insulin from } \\
\text { interstitial space }\end{array}$ & $n_{C}=n_{I} \frac{V_{I}}{V_{Q}}\left(\frac{I_{S S}}{Q_{s s}}-1\right)\left[\mathrm{min}^{-1}\right]$ \\
\hline$u_{e n}(t)$ & Endogenous insulin secretion by pancreas & Variable - Eq. 6 [mU/L/kg/min] \\
\hline$u_{e x}(t)$ & Exogenous insulin therapy & Variable input [mU /min] \\
\hline$V_{P}$ & Fluid volume of plasma & $0.047 \mathrm{~L} / \mathrm{kg}$ \\
\hline$V_{Q}$ & Fluid volume of interstitial space & $V_{Q}=492 \times P N A^{-0.09}-V_{P}[\mathrm{~L} / \mathrm{kg}]$ \\
\hline$\alpha_{G}$ & Saturation on insulin degradation & $0.0 \mathrm{~L} / \mathrm{mU}$ \\
\hline$P N A$ & Post natal age & Known Variable [days] \\
\hline$m_{\text {body }}$ & Body mass & Known Variable [kg] \\
\hline$m_{\text {brain }}$ & Brain mass $\sim 14 \%$ of $m_{\text {body }}[28]$ & Estimated Variable [kg] \\
\hline
\end{tabular}




\subsection{Adaptation of the NICING model for preterm and term infants}

The NICING model of glucose - insulin dynamics was developed for very premature infants, but is sufficiently reflective of premature and term infant physiology for use here. The only points of change were that CNS uptake and interstitial fluid compartment $\left(V_{Q}\right)$ values were allowed to change with gestational age (GA) and post natal age (PNA), by including the literature-based models shown in Figure 2. These two dynamics were adapted based on literature data. Other parameters were assumed similar for term infants, with no known literature data to provide a basis for change.

Only two studies were found exploring CNS uptake of glucose, one of which spanned a range of gestational and postnatal ages. Figure 2a) shows CNS glucose uptake as a function of conceptual gestational age, which is the sum of the gestational age at birth and postnatal age. Older, and likely more developed, infants have greater glucose demand from their CNS, particularly after the 40 week (term) mark. The model here is defined

$$
C N S=0.0031 *(G A+P N A)-0.0338
$$

Extracellular fluid volume is important, as interstitial fluid volume is ECV minus plasma volume. Extracellular fluid volume proportions change with both gestational and postnatal age, as shown in Figure 2b). A literature search of PubMed was performed, expanding on previous work that developed a model for extracellular fluid volume based on postnatal age [24]. Key search terms included plasma, extracellular, interstitial, fluid volume, and neonate/infant, and 6 studies were found $[29,30,31,32$, 33, 34]. Similar to [24], A drop in ECV in the first week following birth is observed across a wide range of studies, and is most prominent in the more premature infants.

An ECV volume model is generated using a linear model derived from [34] to give $\operatorname{ECV}(P N A=0)$ :

$$
E C V_{P N A=0}=-12 \times G A+915
$$


$E C V(P N A>0)$ is described by a proportional drop off based on two long term studies of ECV in very preterm ( 28 weeks) [33] and term ( 40 weeks) [30] infants.

$$
E C V_{\text {frac }}(\mathrm{GA}, \mathrm{PNA})=\operatorname{interp}\left(G A, E C V_{\text {frac }, G A=40}(\mathrm{PNA}), E C V_{\text {frac }, G A=28}(\mathrm{PNA})\right)
$$

Where interp $(x, R X, R Y)$ finds an interpolated solution for $x$ using vectors $\mathrm{RX}$ and $\mathrm{RY}$. The fractional drop in ECV at weeks 28 and 40 are defined:

$$
\begin{aligned}
& E C V_{\text {frac }, G A=28}(\mathrm{PNA})=\operatorname{interp}\left(P N A, P N A_{\text {ref }}=\left[\begin{array}{c}
1 \\
6 \\
13 \\
27 \\
41 \\
63 \\
270 \\
550
\end{array}\right], E C V_{\text {frac }, r e f}=\left[\begin{array}{l}
0.95 \\
0.67 \\
0.64 \\
0.61 \\
0.59 \\
0.58 \\
0.48 \\
0.46
\end{array}\right]\right) \\
& E C V_{\text {frac }, G A=40}(\mathrm{PNA})=\operatorname{interp}\left(P N A, P N A_{\text {ref }}=\left[\begin{array}{c}
1 \\
30 \\
60 \\
120 \\
270 \\
550
\end{array}\right], E C V_{\text {frac }, r e f}=\left[\begin{array}{c}
1 \\
0.90 \\
0.77 \\
0.69 \\
0.61 \\
0.58
\end{array}\right]\right)
\end{aligned}
$$

Where vector values are sourced based on literature values from preterm ( 28 weeks) [33] and term ( 40 weeks) [30] infants.

Finally, the ECV and interstial fluid volume, $V_{Q}$, are computed:

$$
\begin{gathered}
E C V=E C V_{\text {frac },}(\mathrm{GA}, \mathrm{PNA}) \times E C V_{P N A=0} \\
V_{Q}=E C V-V_{P}
\end{gathered}
$$




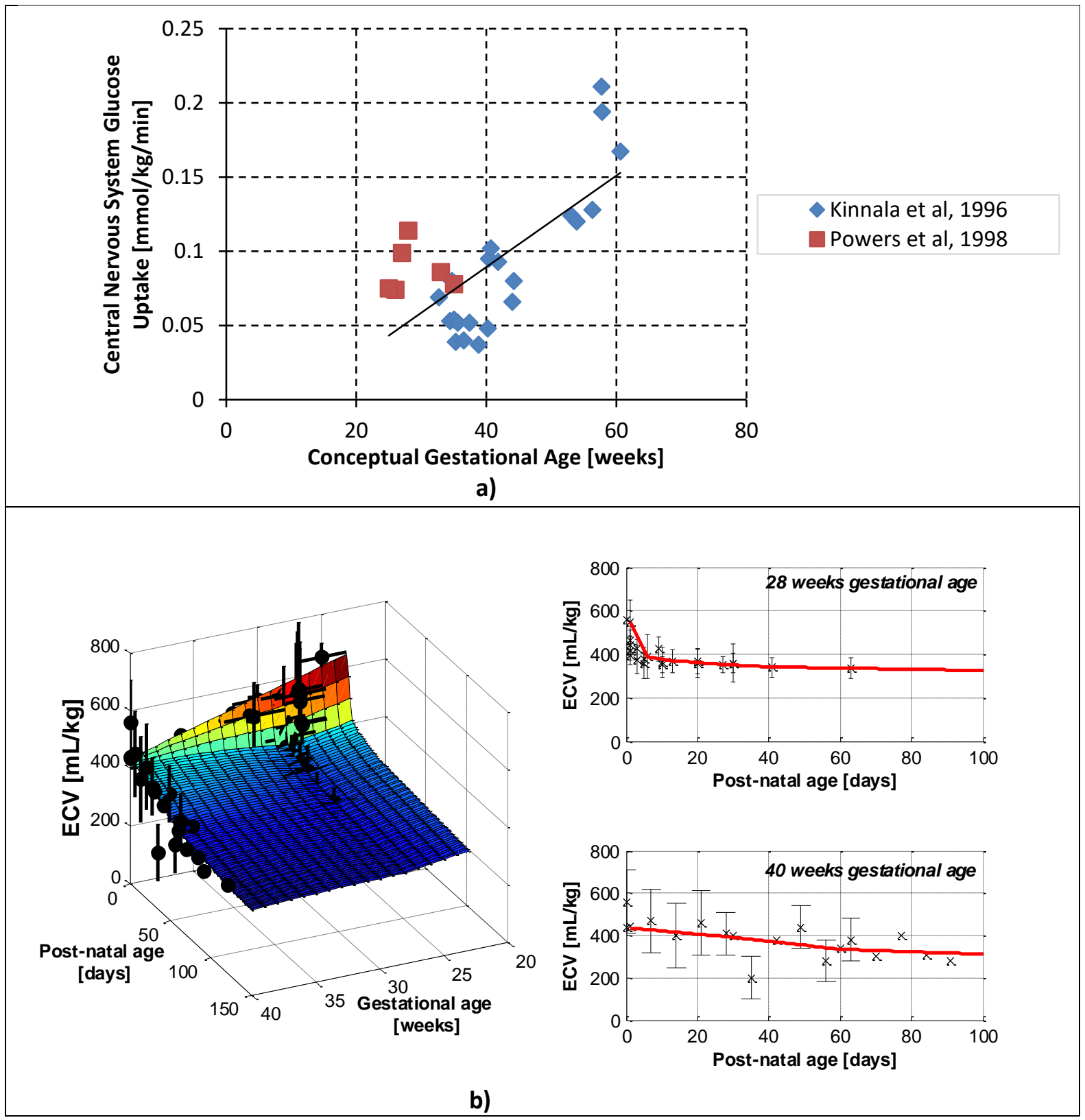

Figure 2: Age-adaptive models of a) CNS uptake based on [35, 36], and b) Extracellular fluid volume (ECV) based on [29, 30, 31, 32, 33]. Conceptual gestational age is the sum of gestational and postnatal age, in weeks. In part $b$, data points reflect literature results, and the surface describes the model used here to determine ECV based on both gestational and postnatal age. 


\subsection{Parameter Identification}

The rate constant of glucose uptake from the gut into the bloodstream, $d_{2}$, was the parameter of interest. Model-based insulin sensitivity $(S I)$ and glucose appearance from the gut can trade off mathematically, so the fitting process was iterative. The parameter $d_{1}$ was left constant as it also trades off with $d_{2}$, and is thus not practically identifiable without further, unavailable, information [37]. Thus, $d_{1}=0.035 \mathrm{~min}^{-1}$ (corresponding to a 20 minute half life) is a reasonably acceptable value based on existing clinical data [38].

The gut model of Equations 1 and 2 was fit to CGM data in conjunction with the NICING model (Equations $3-6)$, using integral based fitting [25]. This process was iterative, so an initial fit using the

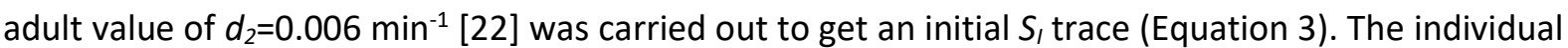
$d_{2}$ values for each feed noted in the clinically charted data were then refit using a grid search (range: 0.001-0.060 $\mathrm{min}^{-1}$ ) to minimise RMS error, based on this SI trace. The range of $d_{2}$ values is chosen to limit the fastest half life of absorption to $\sim 10$ minutes $\left(d_{2}=0.06 \mathrm{~min}^{-1}\right)$. This process was iterated to update the $S_{1}$ trace using the median $d_{2}$ value from the previous iteration. Iterations were continued until $d_{2}$ changed by less than 0.001 ( 3 decimal places, $2-3$ iterations). The time period of CGM data fitted over was nominally 150 min ( 2.5 hours), as this was assumed to approximate the total time for glucose appearance from an enteral feed.

Several factors can confound parameter identification. First, enteral feeds were recorded as having been given 'on the hour,' whereas, in clinical practice, the actual time of feeds is not perfectly time and merely near this mark. For this reason, delivery time of the feed was varied by up to \pm 20 minutes either side of the hour, and the $d_{2}$ value with the lowest fitting error to the associated glucose peak in the CGM data was selected. Second, feed duration is also uncertain, and could vary between 5 and 20 minutes in this cohort depending on volume and clinical preference. Since this time is not recorded delivery duration was assumed as a constant $12 \mathrm{~min}$, and sensitivity of $d_{2}$ to delivery time was evaluated separately. 
The resulting parameter $d_{2}$ was then analysed with respect to PNA, GA, birth weight, and total glucose content of feed to assess any additional demographic trend or dependency to for addition to the model. Statistical comparisons are made, where necessary, using the non-parametric Mann-Whitney test, and $p<0.05$ was considered significant.

\subsection{Clinical Data Cohorts}

Enteral feeds were defined as any manually delivered (syringe/feeding tube) enteral nutritional intake delivered with duration of $\sim 20 \mathrm{~min}$ or less, involving expressed breast milk (EBM), nutritionally fortified EBM, or milk formula. Breastfeeds were not considered due to uncertainty around the total fluid volume delivered, and rate of delivery. Enteral feeds delivered within 2 hours of a breastfeed, a change in parenteral (IV) nutrition, or oral dextrose gel use were not used, to avoid any overlapping glucose appearance.

CGM data from enterally fed infants was available in two cohorts:

Budapest: CGM traces (Guardian REAL-Time, Medtronic Minimed, Northridge, California) from 4 infants receiving 3 hourly enteral breast milk feeds of $20 \mathrm{~mL}$ or more were available from Semmelweis University, Department of Paediatrics, Hungary. Demographic data are in Table 2. According to the local ethical codes this analysis of retrospective CGM data is considered as a clinical data audit, requiring only the depersonalization of the data collected.

SUGAR-BABIES: CGM traces (CGMS system gold, Medtronic Minimed, Northridge, California) from 10 patients from a cohort of 237 infants who were part of the SUGAR-BABIES study [39] were analysed. The SUGAR-BABIES study was approved by the Northern Y Ethics Committee, New Zealand [39]. Selection criteria for the $\mathrm{N}=10$ sample size included patients given 10 or more feeds of $10 \mathrm{~mL}$ or greater volume at least 2 hours apart, with a clear rise and peak in the CGM trace corresponding to the timing of an enteral feed. The sample size $\mathrm{N}=10$ was limited by a pilot data sharing agreement, and patients were randomly chosen from the subset that met the criteria above. The patient cohort is summarised in Table 2. CGM data for the SUGAR-BABIES study data was recalibrated to blood gas measurements 
of BG concentration using the measured current (ISIG) values and method in Signal, Le Compte [40]. Recalibration was not carried out for the Budapest data, as not all necessary data was available.

Table 2: Patient cohort characteristics. Values are median [inter-quartile range].

\begin{tabular}{|c|c|c|}
\hline \multirow{2}{*}{} & \multicolumn{2}{|c|}{ Cohort } \\
\cline { 2 - 3 } & Budapest (N=4) & SUGAR-BABIES (N=10) \\
\hline \hline Gestational Age [wk] & $39[39-40]$ & $36[35-36]$ \\
\hline Birth Weight [kg] & $4.04[3.58-4.65]$ & $2.32[2.14-2.77]$ \\
\hline $\begin{array}{c}\text { Hours after birth received } \\
\text { first feed }\end{array}$ & N/A & $1.6[1.3-4.4]$ \\
\hline $\begin{array}{c}\text { Time after birth CGM was } \\
\text { inserted }\end{array}$ & $22[16-41]$ days & $2.9[0-6.5]$ hours \\
\hline $\begin{array}{c}\text { Enteral feed type } \\
\text { administered } \\
\text { (for samples fitted) }\end{array}$ & Expressed Breast Milk & $\begin{array}{c}\text { Term Formula (N=7) } \\
\text { Preterm Formula (N=1) }\end{array}$ \\
\hline $\begin{array}{c}\text { Per-patient number of feed } \\
\text { episodes recorded on CGM* }\end{array}$ & $42[37-45]$ & $28[21-42]$ \\
\hline $\begin{array}{c}\text { Total number of feed } \\
\text { episodes recorded on CGM* }\end{array}$ & 165 & 318 \\
\hline \begin{tabular}{c} 
Feed volume [mL]* \\
\hline
\end{tabular}
\end{tabular}

*These are feeds that met the selection criteria

\subsection{Reported Absorption Rates for Comparison and Validation}

Glucose absorption rates for short intestinal sections were found in literature. These absorption rates were transformed into 'whole compartment' glucose absorption rates by assuming a linear glucose gradient down the gut, and integrating to give total glucose present in the gut [mmol].

Assuming the glucose concentration profile is roughly linear [41] in the gut, then the glucose concentration profile is:

$$
\breve{P}_{2}=\breve{P}_{2 s}-m x
$$

Where $P_{2 s}$ is the initial glucose concentration. 
Glucose is absorbed over a approximate length of $L g=3 \mathrm{~m}$ [41], and intestinal radius is $r=0.013 \mathrm{~m}$ for adults. Small intestinal length in premature infants gives $L g=0.145 \mathrm{~m}$ and $r=0.007 \mathrm{~m}$ for infants [42]. If at the $L g$ end the glucose concentration is $P_{2 e n d} \approx 0$, then $m$ is defined:

$$
m=\frac{\breve{P}_{2 s}}{L_{g}}
$$

Total glucose absorption from the gut $[\mathrm{mmol} / \mathrm{min}]$ is defined:

$$
\int_{0}^{L s} R_{a b}=d_{2} c P_{2}
$$

Where $P_{2}$ is the total glucose in the gut, and is equal to the integral of the glucose concentration across the gut. The parameter $c$ is the cross sectional area of the gut $\left(c=\pi r^{2}\right)$. Thus, the total absorption across a given gut length, $L s$, of the perfusion study is defined:

$$
\int_{0}^{L s} R_{a b}=d_{2} c \int_{0}^{L s} \breve{P}_{2} d x
$$

Equation 6 can be integrated to give:

$$
\int_{0}^{L s} R_{a b}=d_{2} c\left[\breve{P}_{2 s} L-m \frac{L_{s}^{2}}{2}\right]
$$

To find $d_{2}$, Equation 7 can be rearranged:

$$
d_{2}=\frac{\int_{0}^{L s} R_{a b}}{c\left[\breve{P}_{2 s} L-m \frac{L_{s}^{2}}{2}\right]}
$$

Equation 8 allows estimation of $d_{2}$ from intestinal glucose absorption studies in literature. These values can then be compared to model identified $d_{2}$ values to validate the results based on data from independent clinical studies. 


\subsection{Results}

In the Budapest cohort, a $d_{2}$ solution was converged to in 86 sets of feed data. The median rate constant for glucose uptake from the intestines was $0.014[0.008-0.018] \mathrm{min}^{-1}$, corresponding to an absorption half life of 49 [39 - 86] min. A further 79 feed episodes were discarded after visual inspection of the data due to CGM error (6\%), failure to converge to a solution (12\%), failure of feed to appear in CGM trace (25\%), multiple peaks in expected appearance timeframe causing convergence failure (3\%), or no initial rise in CGM trace during expected appearance time frame (54\%), probably reflecting an earlier than recorded onset of feeding.

For the SUGAR-BABIES cohort, 178 sets of feed data across 10 patients converged to a solution, with a median [IQR] rate constant for glucose uptake of $d_{2}=0.018[0.012-0.026] \mathrm{min}^{-1}$, which corresponds to an absorption half life of 39 [27 - 58] min. In the SUGAR-BABIES cohort a further 140 feed episodes were not used due to failure to converge to a solution (12\%), failure of the feed to appear in the CGM trace (6\%), multiple peaks in CGM data during expected appearance time (11\%), or a lack of initial rise (59\%) or subsequent fall (12\%) in CGM trace around an expected appearance. The latter two cases, totalling $71 \%$ of discarded data, are most probably a result of discrepancy between the recorded feed time and the given feed time of more than 30 minutes, where \pm 20 minutes was assumed in this study.

The identified glucose absorption rate constant for all CGM data from both cohorts was $d_{2}=0.014$ [0.008 - 0.022] $\mathrm{min}^{-1}$, corresponding to an absorption half life of 50 [31 -86] $\mathrm{min}$. In Figure 3 it can be seen that $d_{2}$ is similar between cohorts, despite very different feed volumes (Table 2). Median and IQR of identified $d_{2}$ values varied between patients (Figure 4) with high variability over all patient metrics. Patient 4, who had the lowest rate constant had GA 36 weeks and birth weight $2.8 \mathrm{~kg}$, making no different demographically from the average patient in this cohort. There was no clear trend in $d_{2}$ over PNA $\left(R^{2}<0.001\right)$, BW $\left(R^{2}=0.03\right)$ or feed size (linear $\left.R^{2}=0.03\right)$, where the very low $R^{2}$ values suggest these variables account for little of the variability in the parameter. Thus, there is significant interand intra- patient variability in this parameter. 

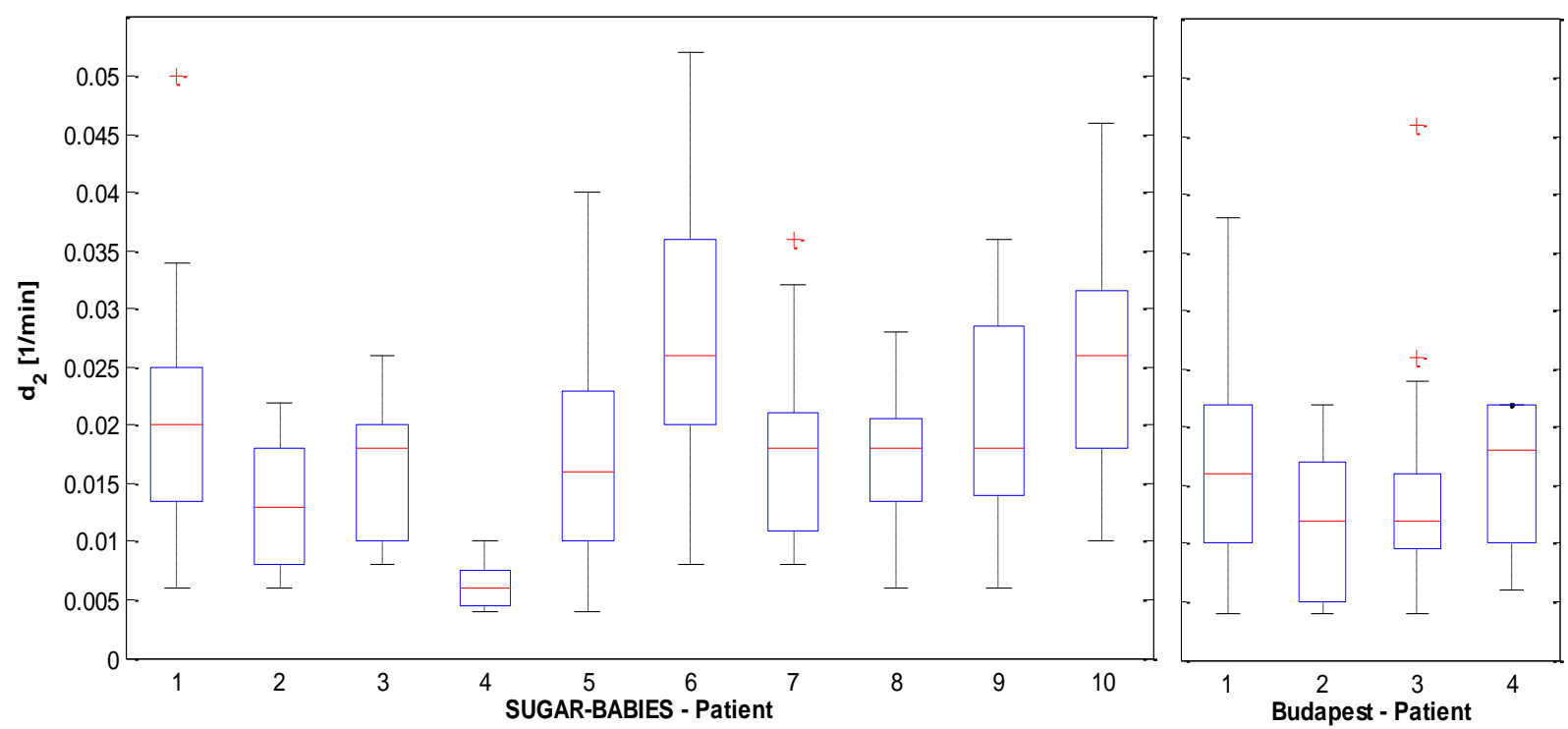

Figure 3: Per patient gut glucose absorption constants (d2) across the SUGAR-BABIES and Budapest cohorts.

In the SUGAR-BABIES data, for breast milk feeds ( $N=57)$, the median absorption rate constant was $d_{2}=$ 0.026 [0.018 - 0.035] $\mathrm{min}^{-1}$ (absorption half life of 27 [20 - 39] $\mathrm{min}$ ) compared to the formula feeds $(\mathrm{N}=118)$ value of $d_{2}=0.018[0.010-0.020] \mathrm{min}^{-1}$ (absorption half life of 39 [34 - 69] $\left.\mathrm{min}\right),(p<0.001)$. This result may reflect differences in gastric emptying (delay in glucose appearance in the gut) with feed type [38]. The remaining 3 feeds were a mixture of breast milk and formula. There is thus a significant difference in glucose processing and absorption based on feed type.

The gut absorption rate constant was, in general, higher with lower gestational age, but the relationship was weak $\left(R^{2}=0.05\right)$, explaining only $5 \%$ of the variability. When the SUGAR-BABIES formula fed data was divided into groups based on tertiles in GA and number of feeds (GA>36, GA = $36, G A>=36, G A<36$ weeks) the gut absorption constant was not significantly different between infants grouped by GA ( $p>=0.06$ over all comparisons). Hence, GA is not a significant model factor.

Figure 4 shows model fit for a representative selection of the converged CGM trace sections from the SUGAR-BABIES cohort. It can be seen that the model achieves a qualitatively good fit in most cases. However, it cannot always completely capture the peak and/or trough of the glucose appearance. In 
addition, there may to be un-modelled appearance dynamics, unrecorded inputs, or sensor error in cases where multiple peaks are not captured.

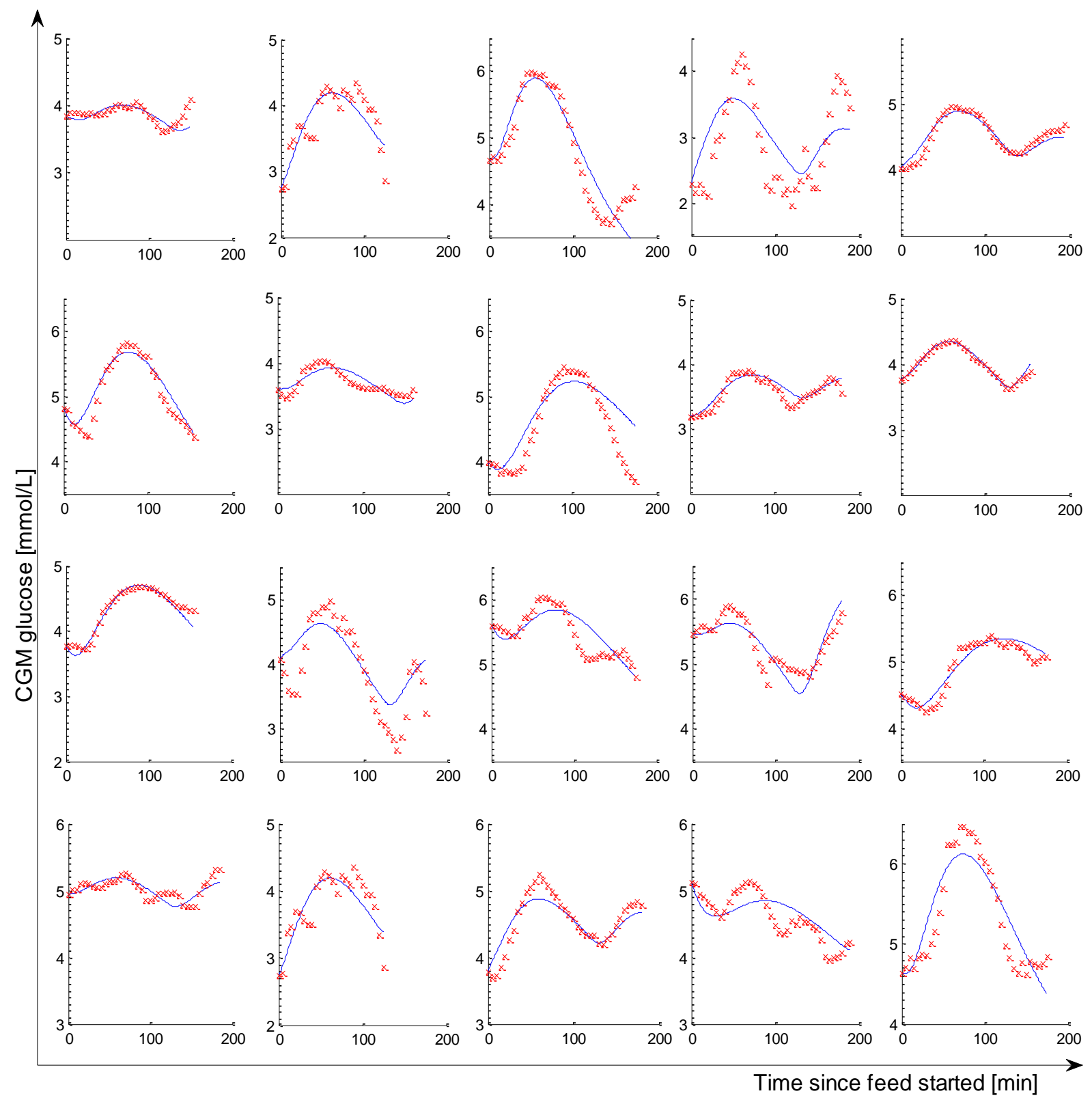

Figure 4: Fitting error over a representative selection of CGM trace sections from SUGAR-BABIES data. The crosses ( $x$ ) show CGM data, and the solid line (-) the model solution around the time of an enteral feed. Time zero is the onset of the feed based on the best model fit. 


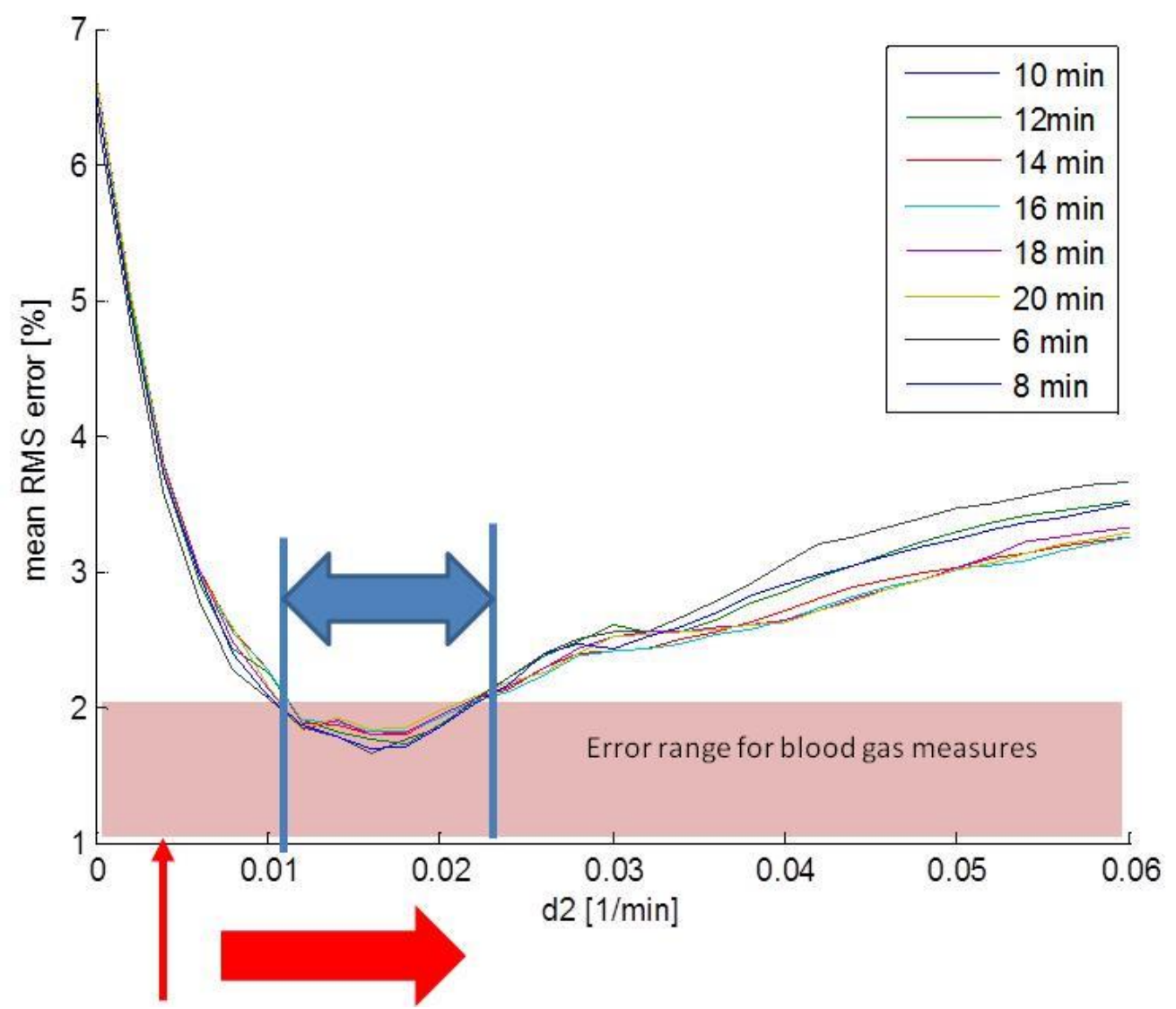

Figure 5: Mean RMS fitting error across the $\mathrm{d} 2$ range for different feed administration periods over the SUGAR-BABIES cohort.

Sensitivity to the time over which the feed was administered was tested, and the results are shown in Figure 5. Fitting error does not differ significantly between different assumed administration times. Given that blood gas measures of glucose have an associated error of $\sim 2 \%$, the optimum cohort-wide $d_{2}$ can be estimated from Figure 5 to lie between $d_{2}=0.011$ and $0.024 \mathrm{~min}^{-1}$, corresponding to a half life of $30-70$ minutes.

\subsection{Comparison to Reported Absorption Rates}

Only two studies explicitly stating the rate of glucose absorption were found in the defined PubMed literature search for both adults [43] and infants [21]. These values were converted from a 'per study gut length' rate to a whole compartment rate constant using Equation 20, and the results are shown in Table 3. The resulting average calculated gut absorption rate constant is higher than the median $d_{2}$ 
values of $0.014-0.018 \mathrm{~min}^{-1}$ identified here using CGM data. However, it is approximately the same order of magnitude.

Table 3: Intestinal glucose absorption rate constants derived from literature measurements of gutabsorption. Glucose infusion $\left(P_{e x}\right)$ rate, initial glucose concentration $\left(P_{2 s}\right)$, and absorption rates $\left(\int_{0}^{L s} R_{a b}\right)$ from literature are used to derive whole compartment absorption rate $\left(\mathrm{d}_{2}\right)$ using Equation 8.

\begin{tabular}{|c|c|c|c|c|c|}
\hline \multirow{17}{*}{ Adults } & Study & $\begin{array}{c}\mathbf{P}_{\mathrm{ex}} \\
{[\mathrm{mmol} / \mathrm{min}]}\end{array}$ & $\begin{array}{c}\mathbf{P}_{2 \mathrm{~s}} \\
{[\mathrm{mmol} / \mathrm{L}]}\end{array}$ & $\begin{array}{c}\int_{0}^{L s} R_{a b} \\
{[\mathrm{mmol} / \mathrm{min} / 25 \mathrm{~cm}]}\end{array}$ & $\mathrm{d}_{2}[1 / \mathrm{min}]$ \\
\hline & \multirow{15}{*}{$\begin{array}{c}\text { Modigliani } \\
\text { and Bernier } \\
\text { [43] }\end{array}$} & 0.5 & 66 & 0.472 & 0.056 \\
\hline & & 1.1 & 133 & 0.728 & 0.043 \\
\hline & & 2.1 & 260 & 0.82 & 0.025 \\
\hline & & 0.7 & 66 & 0.459 & 0.054 \\
\hline & & 1.3 & 133 & 0.763 & 0.045 \\
\hline & & 2.0 & 200 & 0.841 & 0.033 \\
\hline & & 2.6 & 260 & 1.019 & 0.030 \\
\hline & & 1.0 & 66 & 0.585 & 0.069 \\
\hline & & 2.0 & 133 & 0.885 & 0.052 \\
\hline & & 3.0 & 200 & 0.971 & 0.038 \\
\hline & & 3.9 & 260 & 0.999 & 0.030 \\
\hline & & 1.3 & 66 & 0.719 & 0.085 \\
\hline & & 2.7 & 133 & 0.913 & 0.053 \\
\hline & & 4.0 & 200 & 1.091 & 0.042 \\
\hline & & 5.2 & 260 & 1.042 & 0.031 \\
\hline & $\begin{array}{l}\text { Median } \\
\text { [IQR] }\end{array}$ & $\begin{array}{c}2.0 \\
{[1.2-2.8]}\end{array}$ & $\begin{array}{c}133 \\
{[100-230]}\end{array}$ & $\begin{array}{c}0.841 \\
{[0.723-0.985]}\end{array}$ & $\begin{array}{c}0.043 \\
{[0.032-0.054]}\end{array}$ \\
\hline \multirow{5}{*}{$\begin{array}{c}\text { Preterm } \\
\text { Infants } \\
\text { (<37 } \\
\text { weeks) }\end{array}$} & Study & $\begin{array}{c}\text { Pex } \\
{[\mathrm{mmol} / \mathrm{min}]}\end{array}$ & $\begin{array}{c}\mathrm{P}_{2 \mathrm{~s}} \\
{[\mathrm{mmol} / \mathrm{L}]}\end{array}$ & $\begin{array}{c}\int_{0}^{L s} R_{a b} \\
{[\mathrm{mmol} / \mathrm{min} / 15 \mathrm{~cm}]}\end{array}$ & $d_{2}[1 / \mathrm{min}]$ \\
\hline & \multirow{3}{*}{$\begin{array}{c}\text { Shulman } \\
\text { [21] }\end{array}$} & 0.001 & 1 & 0.6 & 0.047 \\
\hline & & 0.01 & 10 & 3.6 & 0.028 \\
\hline & & 0.1 & 100 & 27.9 & 0.022 \\
\hline & Median & 0.01 & 10 & 3.6 & 0.028 \\
\hline
\end{tabular}

The trade-off between gut model transport parameters $d_{1}$ and $d_{2}$ is shown in Figure $6 a$. While there is a global minimum, the parameters trade off significantly within the middle of their likely physiological range. The valleys at either end of the tested range show that so long as at least one dynamic is fast enough to achieve adequate appearance, the system is relatively robust to inter-patient variability in 
absorption constants. The global minimum exists in most cases at the upper end of the $d_{1}$ parameter range, suggesting short gastric emptying times, shorter than reported elsewhere in literature. For example, in Figure 6 a the global minimum occurs at $d_{1}=0.040$, corresponding to a half life of 17 minutes. In contrast, insulin sensitivity and $d_{2}$ trade off to a much smaller degree, as shown in Figure $6 b$, with relatively small change in $S_{1}$ for a relatively large change in $d_{2}$.
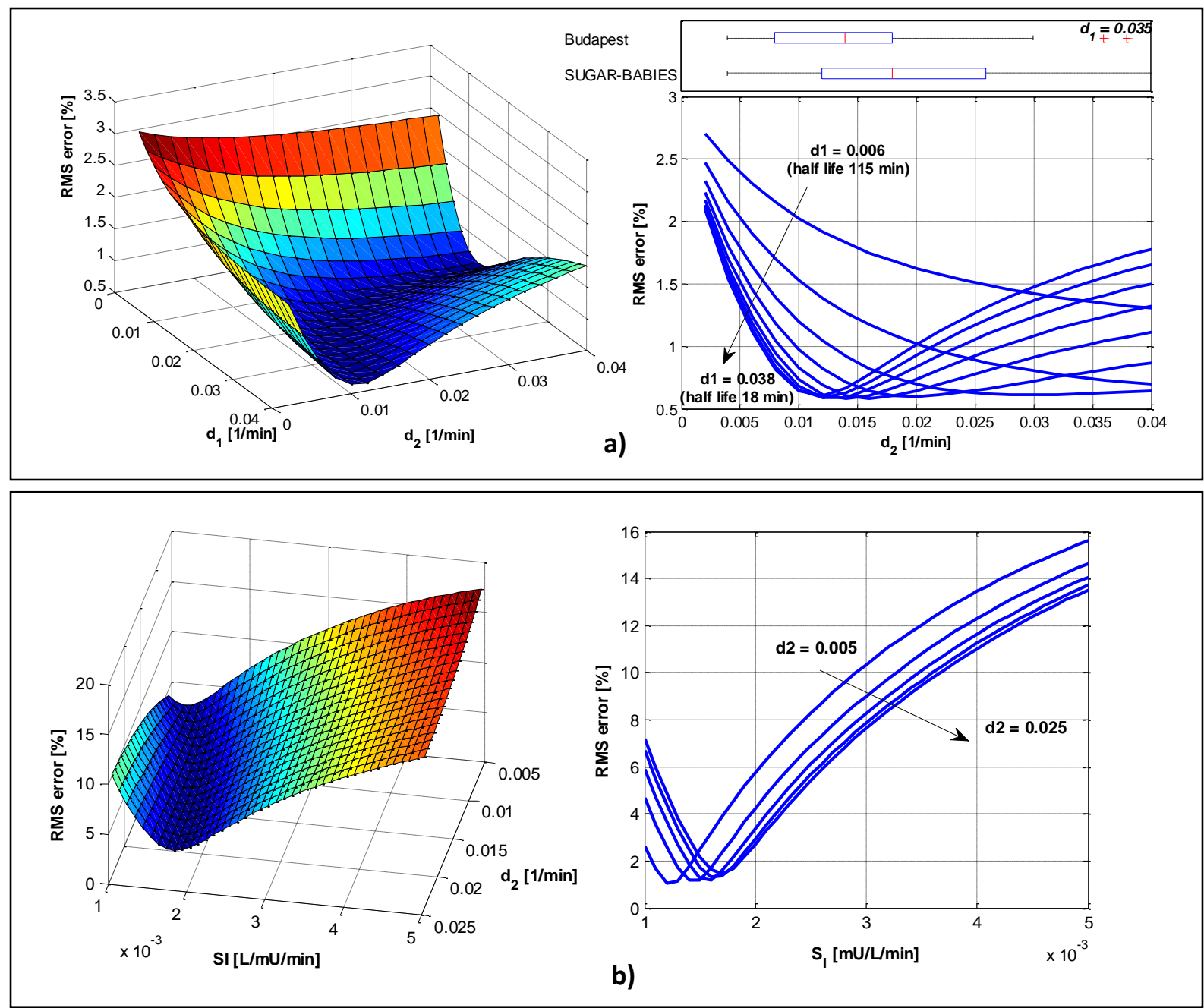

Figure 6: a) A typical error surface showing sensitivity and trade off of a) gastric emptying $\left(d_{1}\right)$ and gut absorption $\left(d_{2}\right)$ parameters, and b) gut absorption $\left(d_{2}\right)$ and insulin sensitivity $\left(S_{1}\right)$ parameters. The waterfall plots in a) and b) have have increments $d_{1}=0.006: 0.004: 0.038[1 / \mathrm{min}]$ and $d_{2}=$ 0.005:0.005:0.025 [1/min] respectively. 


\subsection{Discussion}

The kinetics of glucose absorption are relatively scarce in the premature neonate. While gastric emptying is relatively well studied [38], intestinal glucose absorption is less well studied, and often indirectly assessed. Tracer studies and the appearance of labelled carbon in expired breath has been used to estimate overall lactose absorption from a meal over a 9 hour period [44]. Another study measured mean lactose 'digestion and absorption' to be $\sim 0.7 \mathrm{mg} / \mathrm{min} / \mathrm{cm}$, which if the calculations and assumptions used for glucose absorption are used (Equation 20 in particular with a perfusion rate of $1 \mathrm{~mL} / \mathrm{min}$, a perfusion length of $10 \mathrm{~cm}$, and a perfusate concentration of $70 \mathrm{~g}$ Lactose/L) this corresponds to $\mathrm{d}_{2}=0.009 / \mathrm{min}$.

\subsection{CGM derived results}

CGM data was used to estimate whole gut absorption of glucose in premature infants for a 2 compartment gut model where a delay due to glucose transit of the stomach comprises the first compartment, and the second compartment involves glucose in the gut, from which it is absorbed into the bloodstream. The identified median gut absorption rate constant for intestinal glucose absorption was $d_{2}=0.014 \mathrm{~min}^{-1}$, which corresponds to an absorption half life (time for half of total glucose to be absorbed) of 50 minutes. This value is twice as fast as the half life of 100 minutes used in the adult intensive care case [22].

The median identified gut absorption constant is of the same order as that calculated from adult and infant literature data (0.031 $\mathrm{min}^{-1}$ and $0.028 \mathrm{~min}^{-1}$ respectively, from Table 3), but slightly lower. This outcome seems to indicate the results derived from CGM data are physiologically likely, and that gut absorption in premature and term infants in intensive care may be lower than that in the literature for healthier infants due to their critically ill state, or feed composition differences [38]. Equally, results calculated from literature data are extrapolated from highly controlled studies over short lengths of bowel, and so may not be fully representative of a whole-compartment absorption rate. 
The kinetics of glucose absorption are relatively scarce in the premature neonate. While gastric emptying is relatively well studied [38], intestinal glucose absorption is less well studied, and often indirectly assessed. Thus, while $\mathrm{d} 1 \mathrm{can}$ then be estimated from literature, there is little or no indications of appropriate values for $\mathrm{d} 2$ in literature. This study aimed to estimate this parameter, necessary for accounting for enterally administered nutrition during glycaemic control.

Tracer studies and breath tests have been used to estimate lactose, glucose, or carbohydrate absorption from a meal $[44,45]$. Indirect glucose and galactose absorption was assessed via plasma concentrations of these substrate in another study [46]. As mentioned previously, another study measured mean lactose 'digestion and absorption' to be $\sim 0.7 \mathrm{mg} / \mathrm{min} / \mathrm{cm}$, here was calculated to a corresponding $d_{2}=0.009 / \mathrm{min}$. Other studies have used enzyme activity to measure gut function with varying morbidities and degrees of maturity $[47,48,49,50]$. One study estimated intestinal lactose hydrolysation to glucose and galactose to be approximately $80 \%$ [47]. Overall, almost none of these studies provide data useful for directly quantifying the rate constant for glucose absorption in the intestine. Those that the authors are aware of are given in Table 3.

Gut permeability is a relatively popular topic in literature as it may be related to necrotizing enterocolitis. It seems reasonably well established that gut permeability will increase in the first few post-natal days $[51,52,53]$, but that it decreases with the onset of feeding $[53,54]$, particularly with human milk feeds $[54,55]$. Some studies see increased permeability in infants who have not been enterally fed, similar to an increased permeability ('leakiness') in adults who have been fasted [51]. While in theory increased gut permeability results in higher passive absorption of glucose, Rouwet et. al [51] report general limited capacity of the gut to absorb monosaccharides in very premature infants, with increasing absorptive capacity with increasing postnatal age. Quantitative conclusions from literature are made difficult by the use of surrogate measures and the multiple routes for active and passive absorption of glucose in the gut. 
Counter to expectations, there were no clear and consistent trends in the absorption constant with gestational and post natal age. Enteral feeding of milk causes changes in gut structure and function, even in very premature infants [56]. These changes in gut structure and function include changes in gut motility, enzyme secretion, absorptive capacity, and endocrine response to nutrient absorption [56]. Early enteral feeding has been shown to decrease gut atrophy (breakdown of tissues) and increase intestinal permeability [51], as well as decreasing the incidence of sepsis [57]. However, trends with gestational and postnatal age were not observed, a result that suggests that this method for estimating glucose absorption in the gut is indicative only, and not capable of resolving changes in gut function within $1-3$ days. Equally, changes in gut function over this time may fall within interand intra-patient variability, and these differences may thus be difficult to resolve. Previous studies have observed both increasing and decreasing permeability of the neonatal gut to monosaccharide's during the month after birth [51], and permeability may rise and fall over the first couple of weeks of life [51]. In the absence of consistent models for changes in gut function with gestational and neonatal age, the results derived here are sufficient for model-based control applications.

The practical insignificance of differences between absorption rate constants over GA and between the SUGAR-BABIES data and slightly older Budapest patients with greater feed volume (Table 2 ) is highlighted when blood gas error is considered. Figure 5 shows the RMS error is less than $\sim 2 \%$ between $d_{2}=0.011$ and $0.023 \mathrm{~min}^{-1}$ (half life $30-63 \mathrm{~min}$ ), suggesting a wide valid range from which the glucose absorption can be selected. Almost all median values from groups based on NICU and gestational age fall within this range, thus suggesting any observed differences between groups may result from chance measurement error.

Insulin sensitivity and glucose absorption in the gut trade off to a small degree. However, $S$, is relatively insensitive to changes in the gut absorption constant $d_{2}$ (Figure 6). These results suggest that it is the total glucose absorbed, rather than the rate at which it is absorbed, that has the biggest influence on hourly insulin sensitivity in this clinically validated model. Thus, while a faster $d_{2}$ improves model fit to 
the more measurement dense CGM data, variability in $d_{2}$ does not significantly influence insulin sensitivity, and thus glycaemic control [58].

\subsection{Contributions of Gastric Emptying and Feed Composition}

So long as at least one dynamic is fast enough, $d_{1}$ and $d_{2}$ trade off. As a result, $d_{1}$ was fixed constant, and a gastric emptying half life of 20 minutes $\left(d_{1}=0.035 \mathrm{~min}^{-1}\right)$ was used. This value is within the range of $17-60$ minutes reported in the review by Bourlieu, Menard [38], where high variation in the rate of gastric emptying was seen within and between studies. Some of this variability can be attributed to the different gestational ages and feed types used in across the range of studies. In general, gastric emptying is slower in more premature infants, and is slower in formula fed infants when compared to human breast milk fed infants [38]. Thus, while in this analysis a gastric emptying half life of 20 minutes was appropriate for term and premature infants, a longer half life of 30-45 minutes [38] may be more appropriate in very and/or extremely premature infants.

The breast milk fed SUGAR-BABIES cohort lower glucose absorption half lives, indicating faster absorption of glucose. This result likely reflects the assumption of a constant gastric emptying over the entire cohort. Gastric emptying seems to be dependent on feed types [38], and a relatively faster gut absorption constant in the breast milk fed group could reflect a too fast gastric emptying constant in the formula fed group. However, as fitting error increased with a higher gastric emptying time, it is also possible that milk composition can affect glucose absorption. However, despite this trade off, overall appearance of glucose in the blood from enteral feeds is captured by this model.

In contrast, the glucose absorption rate constant for the breast milk fed Budapest cohort was lower than both the formula fed and breast milk fed cohorts. However, the resultant median and interquartile range for this cohort falls within the $2 \%$ error region of Figure 5 , indicating it may not be possible to separate these effects from CGM sensor noise or noise in blood gas measurements. 
Given the wide range of possible glucose absorption rate constant values within the $2 \%$ error threshold, the study median of $d_{2}=0.0141 / \mathrm{min}$ is a reasonable approximation of glucose absorption dynamics across a wide range of premature infants. This value is also a reasonable approximation of glucose absorption in very premature infants, in lieu of more gestational age specific data. However, since gastric emptying is known to be slower in very premature infants, the gastric emptying rate constant $d_{1}$ can be tailored to this cohort with a value of $d_{1}=0.017 \mathrm{~min}^{-1}$, corresponding to a gastric emptying half life of 40 minutes.

\subsection{Study Limitations}

There are several limitations to this study. First, CGM data is well known for both sensor noise and drift $[59,60,61,62]$. For each feed, the initial glucose value used was the initial CGM BG value, rather than the model fit up to that point, which minimised the effect of sensor drift. In addition, CGM values for the SUGAR-BABIES cohort were recalibrated according to the method of [40], which improves the correlation between sensor data and BG measures. Around $44-48 \%$ of the total feeds in the data failed to converge to a solution or failed to display a clear peak in the CGM data around the time expected based on the clinical feeding record. In addition, additional blood glucose dynamics, probably unrelated to gut function, are evident in some of the examples in Figure 4. As a result, the results in this paper should be considered indicative only of glucose absorption, with glucose absorption being variable over time and between patients, as might be expected in any case.

Additionally, infants were selected for this study primarily based on feed volume and number of feeds. Thus, infants that were likely to display the largest feed related spikes in CGM data were chosen, and so the gut absorption constants found may best reflect relatively large feeds and/or faster absorption, the latter of which might skew model-based results. Table 3 shows that the glucose uptake rate is not in direct proportion to the gut glucose concentration. The results in this paper display no trend and high variability with feed size and glucose concentration, and thus do not confirm, but also, do not rule out, different gut absorption constants that vary with different feed sizes. 
A third potential limitation is the use of the NICING model developed for very low birth weight premature infants, with the premature and term infant data in this study. Fluid volumes and central nervous system uptake were tailored to the infants in this study based on gestational and postnatal age. Without the extremely rare C-peptide and plasma insulin data to tailor insulin clearances and secretion to this older cohort [63], the NICING model was considered to be sufficiently reflective of otherwise similar neonatal physiology for the purposes of this study.

An upper limit on the rate of glucose absorption ( $P_{-}$max in Equation 2) was estimated from that used in an adult ICU model [22] due to a lack of comprehensive and quantitative data for this limit in neonates. As the glucose absorption rates observed in this study are much lower than this upper limit, currently P_max acts as a rarely used limit at the upper end of what might be physiologically expected. This provides consistency and safety in the model-based approach for extreme cases or erroneous data entry, without affecting model dynamics.

In addition, while the calculated absorption rate constants agree broadly with others published in literature, they were calculated within the context of the NICING model, and should thus be used with caution outside of this context. Many of the parameters of the NICING model, which can be found in Table 1, are fixed, and thus the glucose absorption rate constant calculated here may change slightly were these values to change. Parameter sensitivity for the glucose-insulin kinetics with SI has been examined elsewhere $[24,64,65]$. However, given that the glucose absorption constant is predominately a function of the size of glucose appearance peaks, and that no other dynamic in the glucose-insulin model has this shape, parameter trade-off with other NICING model parameter is not expected to be significant.

Finally, the CGM data in this study is from older infants than those the gut absorption model is being designed for. This difference is perhaps partly offset through the use of the NICING model. However, given the lack of such CGM data being available for very or extremely premature infants, the results 
are considered more indicative of premature infant gut glucose absorption than that of adult ICU patients.

Overall, these results are sufficient for an estimation of the gut glucose absorption rate constant, but should be interpreted with caution outside the context of this study and the NICING model. However, they provide a foundation for further specific studies given this is a first modelling attempt with the benefit of CGM data and the results are in line with expected values.

\subsection{Conclusions}

CGM data has been used to estimate the half life, and therefore the rate constant, of glucose absorption from the gut to the bloodstream. The median gut absorption rate constant was $d_{2}=0.014$ $\mathrm{min}^{-1}$, which corresponds to an absorption half life of 50 minutes. There was no significant trend or difference in this constant with gestational or postnatal age, particularly when measurement error in blood glucose is considered. There was a difference between absorption constants in feed formula or breast milk fed infants feed, but this study cannot resolve whether this is due to differences in gastric emptying or gut absorption based, or both. Overall, this paper presents a method for estimating glucose absorption using CGM data for use in a model-based glycaemic control framework.

\subsection{Acknowledgements}

CGM data was provided by $\mathrm{DH}$ and GM, and was analysed independent from the context or studies in which it was originally collected. The main analysis was performed by JD, who was funded by a Canterbury Scholarship. Funding bodies had no involvement in data provision, analysis, or interpretation, or subsequent manuscript preparation or submission. 


\subsection{References}

1. Dweck, H.S. and G. Cassady, Glucose Intolerance in Infants of Very Low Birth Weight. Pediatrics, 1974. 53(2): p. 189.

2. Hays, S.P., B. Smith, and A.L. Sunehag, Hyperglycemia Is a Risk Factor for Early Death and Morbidity in Extremely Low Birth-Weight Infants. Pediatrics, 2006. 118(5): p. 1811-1818.

3. Alaedeen, D.I., M.C. Walsh, and W.J. Chwals, Total parenteral nutrition-associated hyperglycemia correlates with prolonged mechanical ventilation and hospital stay in septic infants. J Pediatr Surg, 2006. 41(1): p. 239-44.

4. Hall, N.J., et al., Hyperglycemia is associated with increased morbidity and mortality rates in neonates with necrotizing enterocolitis. Journal of pediatric surgery, 2004. 39(6): p. 898-901; discussion 898-901.

5. Clark, R.H., P. Thomas, and J. Peabody, Extrauterine growth restriction remains a serious problem in prematurely born neonates. Pediatrics, 2003. 111(5 Pt 1): p. 986-90.

6. American Academy of Pediatrics Committee on Nutrition, Nutritional needs for low-birthweight infants. Pediatrics, 1985. 75: p. 976-986.

7. Cooke, R.J., Nutrient requirements in preterm infants. Pediatr Res, 2003. 53(1): p. 2.

8. Karagol, B.S., et al., Randomized controlled trial of slow vs rapid enteral feeding advancements on the clinical outcomes of preterm infants with birth weight 750-1250 g. JPEN. Journal of parenteral and enteral nutrition, 2013. 37(2): p. 223-8.

9. Collins, J.W., Jr., et al., A controlled trial of insulin infusion and parenteral nutrition in extremely low birth weight infants with glucose intolerance. The Journal of pediatrics, 1991. 118(6): p. 921-7.

10. Pollak, A., et al., Glucose Disposal in Low-Birth-Weight Infants During Steady State Hyperglycemia: Effects of Exogenous Insulin Administration. Pediatrics, 1978. 61(4): p. 546.

11. Vaucher, Y.E., P.D. Walson, and G. Morrow, 3rd, Continuous insulin infusion in hyperglycemic, very low birth weight infants. Journal of pediatric gastroenterology and nutrition, 1982. 1(2): p. 211-7.

12. Ostertag, S., et al., Insulin pump therapy in the very low birth weight infant. Pediatrics, 1986. 78(4): p. 625-630.

13. Thabet, F., et al., Continuous insulin infusion in hyperglycaemic very-low-birth-weight infants receiving parenteral nutrition. Clin Nutr, 2003. 22(6): p. 545-7.

14. Alsweiler, J.M., J.E. Harding, and F.H. Bloomfield, Tight glycemic control with insulin in hyperglycemic preterm babies: a randomized controlled trial. Pediatrics, 2012. 129(4): $p$. 639-47.

15. Beardsall, K., et al., Early Insulin Therapy in Very-Low-Birth-Weight Infants. N Engl J Med, 2008. 359(18): p. 1873-1884.

16. Fisk, L., et al., STAR Development and Protocol Comparison. IEEE Trans Biomed Eng, 2012. 59(12): p. 3357-3364.

17. Le Compte, A.J., et al., Pilot study of a model-based approach to blood glucose control in very-low-birthweight neonates. BMC Pediatr, 2012. 12: p. 117.

18. Le Compte, A.J., et al., Impact of variation in patient response on model-based control of glycaemia in critically ill patients. Comput Methods Programs Biomed, 2013. 109(2): p. 2119.

19. Le Compte, A.J., et al., Blood glucose prediction using stochastic modeling in neonatal intensive care. IEEE Trans Biomed Eng, 2010. 57(3): p. 509-18.

20. Suhaimi, F., et al., What makes tight glycemic control tight? The impact of variability and nutrition in two clinical studies. J Diabetes Sci Technol, 2010. 4(2): p. 284-98.

21. Shulman, R.J., In vivo measurements of glucose absorption in preterm infants. Biology of the neonate, 1999. 76(1): p. 10-8. 
22. Lin, J., et al., A physiological Intensive Control Insulin-Nutrition-Glucose (ICING) model validated in critically ill patients. Comput Methods Programs Biomed, 2011. 102(2): p. 192205.

23. Wong, X., et al., Development of a clinical type 1 diabetes metabolic system model and in silico simulation tool. Journal of Diabetes Science and Technology (JoDST), 2008. 2(3): p. 424435.

24. Dickson, J.L., et al., Insulin kinetics and the Neonatal Intensive Care Insulin-Nutrition-Glucose (NICING) model. Math Biosci, 2017. 284: p. 61-70.

25. Hann, C.E., et al., Integral-based parameter identification for long-term dynamic verification of a glucose-insulin system model. Comput Methods Programs Biomed, 2005. 77(3): p. 259270.

26. Knopp, J.L., et al., Safe and effective glycaemic control in premature infants: observational clinical results from the computerised STAR-GRYPHON protocol. Arch Dis Child Fetal Neonatal Ed, 2018.

27. Coulthard, M.G., Maturation of glomerular filtration in preterm and mature babies. Early Hum Dev, 1985. 11(3-4): p. 281-92.

28. Gunn, C.A., et al., Brain mass estimation by head circumference and body mass methods in neonatal glycaemic modelling and control. Computer methods and programs in biomedicine, 2014. 115(2): p. 47-54.

29. Coran, A.G., R.A. Drongowski, and J.R. Wesley, Changes in total body water and extracellular fluid volume in infants receiving total parenteral nutrition. Journal of pediatric surgery, 1984. 19(6): p. 771-6.

30. Friis-Hansen, B., The extracellular fluid volume in infants and children. Acta paediatrica, 1954. 43(5): p. 444-58.

31. Modi, N., et al., Postnatal weight loss and contraction of the extracellular compartment is triggered by atrial natriuretic peptide. Early human development, 2000. 59(3): p. 201-8.

32. Shaffer, S.G. and V.M. Meade, Sodium balance and extracellular volume regulation in very low birth weight infants. J Pediatr, 1989. 115(2): p. 285-90.

33. Shaffer, S.G., S.K. Bradt, and R.T. Hall, Postnatal changes in total body water and extracellular volume in the preterm infant with respiratory distress syndrome. Journal of Pediatrics, 1986. 109(3): p. 509-14.

34. Avery, G.B., M.A. Fletcher, and M.G. MacDonald, Neonatology : pathophysiology and management of the newborn. 4th ed1994: Philadelphia : Lippincott, c1994.

35. Kinnala, A., et al., Cerebral metabolic rate for glucose during the first six months of life: an FDG positron emission tomography study. Arch Dis Child Fetal Neonatal Ed, 1996. 74(3): p. F153-7.

36. Powers, W.J., et al., Cerebral glucose transport and metabolism in preterm human infants. J Cereb Blood Flow Metab, 1998. 18(6): p. 632-8.

37. Docherty, P.D., et al., A graphical method for practical and informative identifiability analyses of physiological models: a case study of insulin kinetics and sensitivity. Biomed Eng Online, 2011. 10: p. 39.

38. Bourlieu, C., et al., Specificity of infant digestive conditions: some clues for developing relevant in vitro models. Crit Rev Food Sci Nutr, 2014. 54(11): p. 1427-57.

39. Harris, D.L., et al., Dextrose gel for neonatal hypoglycaemia (the Sugar Babies Study): a randomised, double-blind, placebo-controlled trial. Lancet, 2013.

40. Signal, M., et al., Impact of retrospective calibration algorithms on hypoglycemia detection in newborn infants using continuous glucose monitoring. Diabetes technology \& therapeutics, 2012. 14(10): p. 883-90.

41. Borgstrom, B., et al., Studies of intestinal digestion and absorption in the human. The Journal of clinical investigation, 1957. 36(10): p. 1521-36. 
42. Touloukian, R.J. and G.J. Smith, Normal intestinal length in preterm infants. Journal of pediatric surgery, 1983. 18(6): p. 720-3.

43. Modigliani, R. and J.J. Bernier, Absorption of glucose, sodium, and water by the human jejunum studied by intestinal perfusion with a proximal occluding balloon and at variable flow rates. Gut, 1971. 12(3): p. 184-93.

44. Murray, R.D., et al., Comparative absorption of [13C]glucose and [13C]lactose by premature infants. Am J Clin Nutr, 1990. 51(1): p. 59-66.

45. Lifschitz, C.H., Carbohydrate absorption from fruit juices in infants. Pediatrics, 2000. 105(1): p. e4.

46. Okamoto, R., M. Mino, and M. Hayashi, Galactose absorption after oral administration of lactose in neonates. J Nutr Sci Vitaminol (Tokyo), 1989. 35(5): p. 441-50.

47. Kien, C.L., K. Ault, and R.E. McClead, In vivo estimation of lactose hydrolysis in premature infants using a dual stable tracer technique. The American journal of physiology, 1992. 263(5 Pt 1): p. E1002-9.

48. Shulman, R.J., W.W. Wong, and E.O. Smith, Influence of changes in lactase activity and smallintestinal mucosal growth on lactose digestion and absorption in preterm infants. Am J Clin Nutr, 2005. 81(2): p. 472-9.

49. Shulman, R.J., et al., Early feeding, feeding tolerance, and lactase activity in preterm infants. J Pediatr, 1998. 133(5): p. 645-9.

50. Kien, C.L., R.E. McClead, and L. Cordero, Jr., Effects of lactose intake on lactose digestion and colonic fermentation in preterm infants. J Pediatr, 1998. 133(3): p. 401-5.

51. Rouwet, E.V., et al., Intestinal permeability and carrier-mediated monosaccharide absorption in preterm neonates during the early postnatal period. Pediatr Res, 2002. 51(1): p. 64-70.

52. Beach, R.C., et al., Gastrointestinal permeability changes in the preterm neonate. Arch Dis Child, 1982. 57(2): p. 141-5.

53. Colome, G., et al., Intestinal permeability in different feedings in infancy. Acta Paediatr, 2007. 96(1): p. 69-72.

54. Weaver, L.T., et al., Milk feeding and changes in intestinal permeability and morphology in the newborn. J Pediatr Gastroenterol Nutr, 1987. 6(3): p. 351-8.

55. Taylor, S.N., et al., Intestinal permeability in preterm infants by feeding type: mother's milk versus formula. Breastfeed Med, 2009. 4(1): p. 11-5.

56. Aynsley-Green, A., Hormones and postnatal adaptation to enteral nutrition. Journal of pediatric gastroenterology and nutrition, 1983. 2(3): p. 418-27.

57. Flidel-Rimon, O., et al., Early enteral feeding and nosocomial sepsis in very low birthweight infants. Arch Dis Child Fetal Neonatal Ed, 2004. 89(4): p. F289-92.

58. Chase, J.G., et al., Tight glycemic control in critical care - The leading role of insulin sensitivity and patient variability: A review and model-based analysis. Computer Methods and Programs in Biomedicine, 2011. 102(2): p. 156-171.

59. Castle, J.R. and W.K. Ward, Amperometric glucose sensors: sources of error and potential benefit of redundancy. Journal of diabetes science and technology, 2010. 4(1): p. 221-5.

60. Facchinetti, A., et al., Modeling the glucose sensor error. IEEE Trans Biomed Eng, 2014. 61(3): p. 620-9.

61. Facchinetti, A., G. Sparacino, and C. Cobelli, Modeling the error of continuous glucose monitoring sensor data: critical aspects discussed through simulation studies. Journal of diabetes science and technology, 2010. 4(1): p. 4-14.

62. Kuure-Kinsey, M., C.C. Palerm, and B.W. Bequette, A dual-rate Kalman filter for continuous glucose monitoring. Conf Proc IEEE Eng Med Biol Soc, 2006. 1: p. 63-6.

63. Dickson, J.L., et al., Hyperglycaemic preterm babies have sex differences in insulin secretion. Neonatology, 2015. (in press). 
64. Dickson, J.L., et al., On the problem of patient-specific endogenous glucose production in neonates on stochastic targeted glycemic control. J Diabetes Sci Technol, 2013. 7(4): p. 91327.

65. Gunn, C.A., et al., Nasogastric aspiration as an indicator for feed absorption in model-based glycemic control in neonatal intensive care. Journal of diabetes science and technology, 2013. 7(3): p. 717-26. 